PROCEEDINGS OF THE

AMERICAN MATHEMATICAL SOCIETY

Volume 135, Number 11, November 2007, Pages 3677-3684

S 0002-9939(07)08869-7

Article electronically published on May 2, 2007

\title{
VOLUME ENTROPY AND INTEGRAL RICCI CURVATURES OVER CLOSED GEODESICS
}

\author{
SEONG-HUN PAENG
}

(Communicated by Jon G. Wolfson)

\begin{abstract}
We obtain an upper bound of the volume entropy and the simplicial volume with integrals of Ricci curvature over closed geodesics and apply it to the real Schwarz lemma by Besson, Courtois and Gallot.
\end{abstract}

\section{INTRODUCTION}

Let $M$ be an $n$-dimensional compact Riemannian manifold. It is an interesting problem to what extent the geometry and the topology of a closed manifold are determined by some integrals over all closed geodesics. For example, the marked length spectrum $\mathrm{MLS}_{g}$ for a Riemannian metric $g$ is a function which maps a free homotopy class $\langle\gamma\rangle$ of the fundamental group to the length of the shortest geodesic curve in $\langle\gamma\rangle$. In [CD, Croke and Dairbekov showed that if $\mathrm{MLS}_{g_{1}} \geq \mathrm{MLS}_{g_{0}}$, then the area $A\left(g_{i}\right)$ for $g_{i}$ satisfies that $A\left(g_{1}\right) \geq A\left(g_{0}\right)$ in the case of $n=2$. If $A\left(g_{1}\right)=A\left(g_{0}\right)$, then $g_{1}$ is isometric to $g_{0}$. For $n>2$, if $g_{1}=f^{2}(x) g_{0}$ for some smooth positive function $f$, similar results are obtained. In [DS], Dairbekov and Sharafutdinov showed that if $M$ is an Anosov manifold and a smooth function $f$ on $M$ integrates to zero over every closed geodesic, then $f$ must be zero. Furthermore, if $M$ is an Anosov manifold and a smooth 1-form integrates to zero around every closed geodesic, then $f$ is an exact form.

In this paper, we obtain an upper bound of the volume entropy and the simplicial volume with integrals of Ricci curvature over closed geodesics instead of a pointwise curvature bound or an integral curvature on the whole space and then apply it to the real Schwarz lemma by Besson, Courtois and Gallot in BCG1, BCG2], BCG3]. The volume entropy $h(M)$ of $M$ is defined as follows:

$$
h(M)=\lim _{R \rightarrow \infty} \frac{\log (\operatorname{vol}(B(x, R)))}{R},
$$

where $B(x, R)$ is the $R$-ball centered at $x$ in $\tilde{M}$. If the Ricci curvature is bounded below, we obtain an upper bound of the volume entropy by the Bishop-Gromov volume comparison theorem.

Received by the editors July 20, 2006 and, in revised form, August 7, 2006.

2000 Mathematics Subject Classification. Primary 53C20; Secondary 53C23.

Key words and phrases. Volume entropy, simplicial volume, integral Ricci curvature, hyperbolic manifold.

This work was supported by grant No. R01-2006-000-10047-0(2006) from the Basic Research Program of the Korea Science \& Engineering Foundation. 
We assume that $K_{M}<0$ and obtain an upper bound of the entropy depending on integrals of Ricci curvature over closed geodesics. First, we introduce the following notation for the integral of Ricci curvature over a geodesics: Let $g(x)$ be the smallest eigenvalue of the Ricci tensor at $x \in M$ and let $u_{+}=\max (0, u)$ be the positive part of $u$. We denote the function $\left((-g(x)+(n-1) \lambda)_{+}\right)$by $\rho(x)$. We can consider the function $\rho$ on $M$ as a function on the unit tangent bundle $U M$, i.e. $\rho\left(v_{x}\right)=\rho(x)$. Let $\xi_{t}$ be the geodesic flow of $M$. If $K_{M}<0$, then the geodesic flow on $U M$ is ergodic. We define a function $\bar{\rho}_{+}$as follows:

$$
\bar{\rho}_{+}\left(w_{y}\right)=\lim _{R \rightarrow \infty} \frac{1}{R} \int_{0}^{R} \rho\left(\xi_{t}\left(w_{y}\right)\right)^{p} d t .
$$

By the Birkhoff ergodic theorem $[\mathrm{KH}$, we have

$$
\bar{\rho}_{+}\left(w_{y}\right)=\int_{v_{x} \in U M} \rho\left(v_{x}\right)^{p} d \mu=\int_{S^{n-1}} \int_{M} \rho(x)^{p} d v d \theta
$$

for almost every $w_{y} \in U M$, where $\mu$ is the Liouville measure which is invariant with respect to the geodesic flow $\left[\mathrm{C}\right.$. Hence $\bar{\rho}_{+}$is constant almost everywhere. However $\bar{\rho}_{+}$is not only nonconstant but also discontinuous in general.

If $w_{y}$ and $v_{x}$ in $U M$ are contained in the same orbit of the geodesic flow, i.e. $w_{y}$ and $v_{x}$ are velocity vectors of the same geodesic, then $\bar{\rho}_{+}\left(w_{y}\right)=\bar{\rho}_{+}\left(v_{x}\right)$. Hence $\bar{\rho}_{+}$ can be considered as a function from the set of all closed geodesics $\Gamma$ to $\mathbb{R}$. Then

$$
\bar{\rho}_{+}(\gamma)=\frac{1}{\operatorname{Length}(\gamma)} \int_{\gamma} \rho^{p} .
$$

We define $k_{\Gamma}(\lambda, p)$ as follows:

$$
k_{\Gamma}(\lambda, p)=\sup \left\{\bar{\rho}_{+}(\gamma) \mid \gamma \in \Gamma\right\} .
$$

If $\operatorname{Ric}_{M} \geq(n-1) \lambda$, then $k_{\Gamma}(\lambda, p)=0$.

Our main theorem is as follows.

Theorem 1.1. Let $M$ be an $n$-dimensional compact Riemannian manifold with the sectional curvature $K_{M}<0$. For $p>\frac{1}{2}$, the volume entropy of $M$ satisfies that

$$
h(M) \leq(n-1) \sqrt{|\lambda|}+\sqrt{n-1} k_{\Gamma}(\lambda, p)^{\frac{1}{2 p}} .
$$

We don't need to assume $2 p>n$ unlike as in PW1. From Theorem 1.1, we obtain an upper bound on the simplicial volume. Gromov proved the following inequality between the volume entropy and the simplicial volume $\|M\|$ of $M$ ([G2], BCG2 ):

$$
h(M)^{n} C_{n} n ! \operatorname{vol}(M) \geq\|M\|,
$$

so it was obtained that if $\operatorname{Ric}_{M} \geq-1 /(n-1)$, then

$$
\|M\| \leq C_{n} n ! \operatorname{vol}(M)
$$

where $C_{n}=\Gamma\left(\frac{n}{2}\right) /\left(\sqrt{\pi} \Gamma\left(\frac{n+1}{2}\right)\right)$. As an immediate consequence of [G2], BCG2], we have the following corollary:

Corollary 1.2. Let $M$ be an n-dimensional compact Riemannian manifold with $K_{M}<0$. The simplicial volume $\|M\|$ of $M$ satisfies that

$$
\|M\| \leq C_{n} n !\left((n-1) \sqrt{|\lambda|}+\sqrt{n-1} k_{\Gamma}(\lambda, p)^{\frac{1}{2 p}}\right)^{n} \operatorname{vol}(M) .
$$

From the real Schwarz lemma [BCG3, we obtain the following corollary: 
Corollary 1.3. Let $M$ and $N$ be $n$-dimensional compact negatively curved manifolds for $n \geq 3$.

(a) If $K_{N} \leq \lambda<0$, then any continuous map $f: M \rightarrow N$ can be deformed to a smooth map $F$ such that

$$
\operatorname{vol}(F(A)) \leq\left(1+\frac{k_{\Gamma}(\lambda, p)^{\frac{1}{2 p}}}{\sqrt{(n-1)|\lambda|}}\right)^{n} \operatorname{vol}(A) .
$$

(b) If $\operatorname{vol}(M) \leq \operatorname{vol}(N)$ and

$$
K_{N}<-\left(\sqrt{|\lambda|}+\frac{k_{\Gamma}(\lambda, p)^{\frac{1}{2 p}}}{\sqrt{n-1}}\right)^{2}
$$

then there exists no continuous map from $M$ to $N$ of nonzero degree.

In Section 2, we review the real Schwarz lemma in BCG3. In Section 3, we will prove Theorem 1.1 and Corollary 1.3.

\section{ReAl SChWARZ LEMma}

We review the real Schwarz lemma BCG3.

Theorem 2.1. Let $M, N$ be real complete Riemannian manifolds such that $3 \leq$ $\operatorname{dim}(M) \leq \operatorname{dim}(N)$. Let us assume that there exists some constant $C \neq 0$ such that $K_{N} \leq-C^{2}$ and $\operatorname{Ric}_{M} \geq-(n-1) C^{2}$. Then any continuous map $f: M \rightarrow N$ may be deformed to a family of $C^{1}$ maps $F_{\delta}$ for $\delta \rightarrow 0$ such that $\operatorname{vol}\left(F_{\delta}(A)\right) \leq(1+\delta) \operatorname{vol}(A)$ for any measurable set $A$ in $M$. Moreover, assume that $M$ and $N$ are compact.

(a) If $\operatorname{vol}(M)=|\operatorname{deg} f| \operatorname{vol}(N)$, then $M$ and $N$ have constant sectional curvature $-C^{2}$ and we may choose $F_{\delta}$ 's such that they converge to a Riemannian covering $F$ as $\delta \rightarrow 0$.

(b) If $M, N$ are homotopically equivalent and $K_{M}<0$, then any homotopy equivalence $f$ may be deformed to a smooth map $F$ such that $\operatorname{vol}(F(A)) \leq \operatorname{vol}(A)$ for any open subset $A$ in $M$, the equality being attained if and only if $F$ is an isometry on $A$.

Immediately, we obtain the following corollary:

Corollary 2.2. Let $M, N$ be $n$-dimensional compact Riemannian manifolds for $n \geq 3$. Let $K_{N} \leq-C^{2}$ and $\operatorname{Ric}_{M} \geq-(n-1) \Lambda^{2}$ for $\Lambda<C$. If $\operatorname{vol}(N) \geq \operatorname{vol}(M)$, then there exists no continuous map from $M$ to $N$ of nonzero degree.

Proof. Assume that $K_{N} \leq-C^{2}, \operatorname{Ric}_{M} \geq-(n-1) \Lambda^{2}$ for $\Lambda<C$ and $\operatorname{vol}(N) \geq$ $\operatorname{vol}(M)$. Rescaling the metric of $M$ such that $\operatorname{Ric}_{M} \geq-(n-1) C^{2}$, then $\operatorname{vol}(M)<$ $\operatorname{vol}(N)$. From Theorem 2.1, if there is a continuous map from $M$ to $N$ of nonzero degree, then $\operatorname{vol}(N) \leq \operatorname{vol}(M)$, which is a contradiction. Hence there is no continuous map from $M$ to $N$ of nonzero degree.

\section{Proof of MAIN THEOREM}

We will follow the notation in PW1. Around $x \in M$, we use polar coordinates and write the volume element as $d \mathrm{vol}=\omega d t \wedge d \theta_{n-1}$, where $d \theta_{n-1}$ is the standard volume element on the unit sphere $S^{n-1}$. We define $\omega$ to be 0 beyond the cut locus. Then we have

$$
\omega^{\prime}=h \omega,
$$


where $h$ is the mean curvature of the distance spheres around $x$, and $h$ satisfies that

$$
h^{\prime}+\frac{h^{2}}{n-1} \leq-\operatorname{Ric}\left(\partial_{t}, \partial_{t}\right)
$$

where $\partial_{t}$ is the unit gradient of the distance function.

In the $n$-dimensional space form $S_{\lambda}^{n}$ of constant curvature $\lambda$, we obtain $\omega_{\lambda}$ and $h_{\lambda}$ for $S_{\lambda}$ similarly as $\omega$ and $h$, respectively. Then

$$
h_{\lambda}^{\prime}+\frac{h_{\lambda}^{2}}{n-1}=-(n-1) \lambda .
$$

Let $\psi=\psi(t, \cdot)=\max \left\{0, h(t, \cdot)-h_{\lambda}(t, \cdot)\right\}$. Since $\frac{\omega^{\prime}}{\omega}=h$, integrating this equation, we obtain that

$$
\log \left(\frac{\omega(R)}{\omega(r)}\right)=\int_{r}^{R} h d t \leq \int_{r}^{R} h_{\lambda} d t+\int_{0}^{R} \psi d t .
$$

Then we have

$$
\omega(R) \leq e^{\int_{0}^{R} \psi d t} e^{\int_{r}^{R} h_{\lambda} d t} \omega(r)
$$

As $r \rightarrow 0, \omega_{\lambda}(r) / \omega(r) \rightarrow 1$, so we have

$$
\lim _{r \rightarrow 0} e^{\int_{r}^{R} h_{\lambda} d t} \omega(r)=\omega_{\lambda}(R) .
$$

Then for $\lambda<0$,

$$
\omega(R) \leq e^{\int_{0}^{R} \psi d t} \omega_{\lambda}(R) \leq A_{n} e^{(n-1) \sqrt{|\lambda|} R+\int_{0}^{R} \psi d t}
$$

for a constant $A_{n}$ and for $\lambda=0$,

$$
\omega(R) \leq e^{\int_{0}^{R} \psi d t} \omega_{\lambda}(R) \leq \frac{\omega_{n-1}}{n} R^{n} e^{\int_{0}^{R} \psi d t},
$$

where $\omega_{n-1}$ is the volume of a standard $(n-1)$-sphere.

We will prove the following lemma similarly as Lemma 2.2 in [PW1]:

Lemma 3.1. For any $p>\frac{1}{2}$, we have

$$
\int_{0}^{r} \psi^{2 p} d t \leq(n-1)^{p} \int_{0}^{r} \rho^{p} d t
$$

Proof. We have

$$
\psi^{\prime}+\frac{\psi^{2}}{n-1}+\frac{2 \psi h_{\lambda}}{n-1} \leq \rho .
$$

Multiply by $\psi^{2 p-2}$ and integrate to get

$$
\int_{0}^{r} \psi^{\prime} \psi^{2 p-2} d t+\frac{1}{n-1} \int_{0}^{r} \psi^{2 p} d t+\frac{2}{n-1} \int_{0}^{r} h_{\lambda} \psi^{2 p-1} d t \leq \int_{0}^{r} \rho \psi^{2 p-2} d t
$$

Since $\left(\psi^{2 p-1}\right)^{\prime}=(2 p-1) \psi^{\prime} \psi^{2 p-2}$,

$$
\int_{0}^{r} \psi^{\prime} \psi^{2 p-2} d t=\left.\frac{1}{2 p-1} \psi^{2 p-1}\right|_{0} ^{r} \geq 0
$$

Inserting this in (3.11), we obtain that

$$
\frac{1}{n-1} \int_{0}^{r} \psi^{2 p} d t+\frac{2}{n-1} \int_{0}^{r} h_{\lambda} \psi^{2 p-1} d t \leq \int_{0}^{r} \rho \psi^{2 p-2} d t
$$


which implies that

$$
\begin{aligned}
\frac{1}{n-1} \int_{0}^{r} \psi^{2 p} d t & \leq \int_{0}^{r} \rho \psi^{2 p-2} d t \\
& \leq\left(\int_{0}^{r} \rho^{p} d t\right)^{\frac{1}{p}}\left(\int_{0}^{r} \psi^{2 p} d t\right)^{1-\frac{1}{p}} .
\end{aligned}
$$

Dividing by $\left(\int_{0}^{r} \psi^{2 p} d t\right)^{1-\frac{1}{p}}$, then we obtain that

$$
\frac{1}{n-1}\left(\int_{0}^{r} \psi^{2 p} d t\right)^{\frac{1}{p}} \leq\left(\int_{0}^{r} \rho^{p} d t\right)^{\frac{1}{p}}
$$

Consequently, we have

$$
\int_{0}^{r} \psi^{2 p} d t \leq(n-1)^{p} \int_{0}^{r} \rho^{p} d t
$$

which completes the proof of Lemma 3.1.

In the proof of Lemma 2.2 in [PW1, it is an essential condition that $p>n / 2$, but our lemma holds for $p>\frac{1}{2}$.

If the convergence (1.1) is uniform with respect to $R$, then we obtain an upper bound of the volume of $R$-balls for sufficiently large $R$ by (3.5) and Lemma 3.1. The sequence (1.1) does not converge everywhere but almost everywhere, so generally we cannot have the uniform convergence. But if we assume that $K_{M}<0$, then we can prove the following lemmas.

Lemma 3.2. For every $v_{x} \in U M$, we have

$$
\lim _{R \rightarrow \infty} \frac{1}{R} \int_{0}^{R} \rho\left(\xi_{t}\left(v_{x}\right)\right)^{p} d t \leq k_{\Gamma}(\lambda, p)
$$

Proof. For any $v_{x} \in U M$, let $\tilde{v}$ and $\tilde{x}$ be the lifting of $v_{x}$ and $x$ to $\tilde{M}$, respectively, and let $\gamma_{v}$ be the geodesic ray from $\tilde{x}$ such that $\gamma_{v}^{\prime}(0)=\tilde{v}$. Let $D_{n}$ be the fundamental domain in the universal covering space that contains $\gamma_{v}(n)$, and let $x(n)$ be the deck transformation of $x$ in $D_{n}$. Let $\gamma_{n}$ be the geodesic segment from $x$ to $x(n)$. Then it is projected to a closed curve by the covering map $\Pi$. Let $\bar{\gamma}_{n}$ be the shortest closed geodesic homotopic to $\Pi\left(\gamma_{n}\right)$. Then the tangent vectors of $\bar{\gamma}_{n}$ make a closed orbit of the geodesic flow in $U M$. Let $\bar{x}_{n}$ be $\bar{\gamma}_{n}(0)$ and $\tilde{\gamma}_{n}$ be the lifting of $\bar{\gamma}_{n}$ to the universal covering space such that $\tilde{\gamma}_{n}(0) \in D_{0}$. There exists a geodesic segment $\alpha_{n}$ from $\tilde{x}_{n}=\tilde{\gamma}_{n}(0)$ to $\gamma_{v}(n)$. Since $\bar{\gamma}_{n}$ is a closed geodesic, we have

$$
\lim _{n \rightarrow \infty} \frac{1}{n} \int_{0}^{n} \rho\left(\xi_{t}\left(\tilde{\gamma}_{n}^{\prime}(0)\right)\right)^{p} d t=\lim _{n \rightarrow \infty} \frac{1}{n} \int_{0}^{n} \rho\left(\tilde{\gamma}_{n}^{\prime}(t)\right)^{p} d t \leq k_{\Gamma}(\lambda, p)
$$

from our assumption. We have $d\left(x(n), \tilde{\gamma}_{n}(n)\right) \leq 2 D$ for the diameter $D$ of $M$ since $\bar{\gamma}_{n}$ and $\Pi\left(\gamma_{n}\right)$ are homotopic. Since $K_{M}<0$,

$$
\int_{0}^{n} d\left(\alpha_{n}(t), \tilde{\gamma}_{n}(t)\right) d t \leq C_{1}(M)
$$

for some constant $C_{1}(M)$ depending on $M$. (Note that the areas of any triangles are bounded if $K_{M}<0$.) Also since $\rho^{p}$ is a Lipschitz function on $M$, we obtain 
that

$$
\begin{aligned}
& \int_{0}^{n}\left|\rho\left(\alpha_{n}^{\prime}(t)\right)^{p}-\rho\left(\tilde{\gamma}_{n}^{\prime}(t)\right)^{p}\right| d t \leq C_{2}(M), \\
& \int_{0}^{n}\left|\rho\left(\alpha_{n}^{\prime}(t)\right)^{p}-\rho\left(\gamma_{v}^{\prime}(t)\right)^{p}\right| d t \leq C_{2}(M),
\end{aligned}
$$

where $C_{2}(M)$ is a constant depending on $M$. Then we have

$$
\int_{0}^{n}\left|\rho\left(\gamma_{v}^{\prime}(t)\right)^{p}-\rho\left(\tilde{\gamma}_{n}^{\prime}(t)\right)^{p}\right| d t \leq 2 C_{2}(M)
$$

and

$$
\begin{aligned}
\lim _{n \rightarrow \infty} \frac{1}{n} \int_{0}^{n} \rho\left(\xi_{t}\left(v_{x}\right)\right)^{p} d t & =\lim _{n \rightarrow \infty} \frac{1}{n} \int_{0}^{n} \rho\left(\gamma_{v}^{\prime}(t)\right)^{p} d t \\
& =\lim _{n \rightarrow \infty} \frac{1}{n} \int_{0}^{n} \rho\left(\tilde{\gamma}_{n}^{\prime}(t)\right)^{p} d t \\
& \leq k_{\Gamma}(\lambda, p),
\end{aligned}
$$

which completes the proof.

Lemma 3.3. For any fixed $\epsilon>0$, there exists $R>0$ such that

$$
\frac{1}{R} \int_{0}^{R} \rho\left(\xi_{t}\left(v_{x}\right)\right)^{p} d t<(1+\epsilon) k_{\Gamma}(\lambda, p)
$$

for every $v_{x} \in U M$.

Proof. Let

$$
\mathcal{R}\left(r, v_{x}\right)=\frac{1}{r} \int_{0}^{r} \rho\left(\xi_{t}\left(v_{x}\right)\right)^{p} d t .
$$

Then $\mathcal{R}\left(r, v_{x}\right)$ is a continuous function for $r>0$ and $v_{x} \in U M$. So the set

$$
V=\left\{\left(r, v_{x}\right) \mid \mathcal{R}\left(r, v_{x}\right)<(1+\epsilon) k_{\Gamma}(\lambda, p)\right\}
$$

is an open set for a small $\epsilon>0$. From Lemma 3.2, for every $v_{x} \in U M$, there exists $T>0$ such that if $r \geq T$, then

$$
\frac{1}{r} \int_{0}^{r} \rho\left(\xi_{t}\left(v_{x}\right)\right)^{p} d t<(1+\epsilon) k_{\Gamma}(\lambda, p)
$$

Let

$$
T\left(v_{x}\right)=\inf \left\{T>0 \mid \frac{1}{r} \int_{0}^{r} \rho\left(\xi_{t}\left(v_{x}\right)\right)^{p} d t<(1+\epsilon) k_{\Gamma}(\lambda, p) \text { for any } r>T\right\} .
$$

Then the set

$$
V_{\infty}=\left\{\left(r, v_{x}\right) \mid r>T\left(v_{x}\right)\right\}
$$

is a component of $V$ containing infinity. So $V_{\infty}$ is also open. If $T\left(v_{x}\right)$ is not upper semi-continuous, then $V_{\infty}$ is not open, so $T$ is upper semi-continuous. Since $U M$ is compact and $T$ is upper semi-continuous, $T(U M)$ is bounded. So there exists $R$ such that

$$
\frac{1}{R} \int_{0}^{R} \rho\left(\xi_{t}\left(v_{x}\right)\right)^{p} d t<(1+\epsilon) k_{\Gamma}(\lambda, p)
$$

for every $v_{x} \in U M$. 
Proof of Theorem 1.1. By the Hölder inequality, we have

$$
\begin{aligned}
\frac{1}{R} \int_{0}^{R} \psi d t & \leq \frac{1}{R}\left(\int_{0}^{R} \psi^{2 p} d t\right)^{\frac{1}{2 p}} R^{\frac{1}{q}} \\
& \leq \frac{1}{R}\left((n-1)^{p} \int_{0}^{R} \rho^{p} d t\right)^{\frac{1}{2 p}} R^{\frac{1}{q}} \\
& \leq \sqrt{n-1}\left(\frac{1}{R} \int_{0}^{R} \rho^{p} d t\right)^{\frac{1}{2 p}}
\end{aligned}
$$

for $1 /(2 p)+1 / q=1$. For a fixed $\epsilon>0$, we have

$$
\begin{aligned}
\frac{1}{R} \int_{0}^{R} \psi d t & \leq \sqrt{n-1}\left(\frac{1}{R} \int_{0}^{R} \rho^{p} d t\right)^{\frac{1}{2 p}} \\
& \leq \sqrt{n-1}\left((1+\epsilon) k_{\Gamma}(\lambda, p)\right)^{\frac{1}{2 p}}
\end{aligned}
$$

from Lemma 3.3. For $\lambda<0$, we obtain from (3.7) that

$$
\begin{aligned}
\int_{S^{n-1}} \int_{0}^{R} \omega d t d \theta_{n-1} & \leq \int_{S^{n-1}} \int_{0}^{R} A_{n} e^{(n-1) \sqrt{|\lambda|} R+\int_{0}^{R} \psi d s} d t d \theta_{n-1} \\
& =A_{n} R e^{(n-1) \sqrt{|\lambda|} R} \int_{S^{n-1}} e^{\int_{0}^{R} \psi d s} d \theta_{n-1} \\
& \leq A_{n} R e^{(n-1) \sqrt{|\lambda|} R} e^{\sqrt{n-1} R\left((1+\epsilon) k_{\Gamma}(\lambda, p)\right)^{\frac{1}{2 p}}} \omega_{n-1}
\end{aligned}
$$

for some constant $A_{n}$. Hence

$$
\begin{aligned}
\lim _{R \rightarrow \infty} \frac{1}{R} \log (\operatorname{vol}(B(y, R))) & =\frac{1}{R} \log \left(\int_{S^{n-1}} \int_{0}^{R} \omega d t d \theta_{n-1}\right) \\
& \leq(n-1) \sqrt{|\lambda|}+\sqrt{n-1}\left((1+\epsilon) k_{\Gamma}(\lambda, p)\right)^{\frac{1}{2 p}} .
\end{aligned}
$$

For $\lambda=0$, by (3.8) we have

$$
\begin{aligned}
\int_{S^{n-1}} \int_{0}^{R} \omega d t d \theta_{n-1} & \leq \int_{S^{n-1}} \int_{0}^{R} \frac{\omega_{n-1}}{n} R^{n} e^{\int_{0}^{R} \psi d s} d t d \theta_{n-1} \\
& =R^{n+1} \frac{\omega_{n-1}}{n} \int_{S^{n-1}} e^{\int_{0}^{R} \psi d s} d \theta_{n-1} \\
& \leq R^{n+1} \frac{\omega_{n-1}}{n} e^{\sqrt{n-1} R\left((1+\epsilon) k_{\Gamma}(\lambda, p)\right)^{\frac{1}{2 p}}} \omega_{n-1}
\end{aligned}
$$

Hence we have

$$
\lim _{R \rightarrow \infty} \frac{1}{R} \log (\operatorname{vol}(B(y, R))) \leq \sqrt{n-1}\left((1+\epsilon) k_{\Gamma}(\lambda, p)\right)^{\frac{1}{2 p}} .
$$

Since $\epsilon$ is arbitrary, we have from (3.21) and (3.23) that

$$
\begin{aligned}
h(M) & =\lim _{R \rightarrow \infty} \frac{1}{R} \log (\operatorname{vol}(B(y, R))) \\
& \leq(n-1) \sqrt{|\lambda|}+\sqrt{n-1} k_{\Gamma}(\lambda, p)^{\frac{1}{2 p}}
\end{aligned}
$$

for $\lambda \leq 0$, which completes the proof of Theorem 1.1. 
Proof of Corollary 1.3. For the proof of Corollary 1.3, we only need to apply the proof of the real Schwarz lemma BCG3] to our case. Actually, the condition that $\operatorname{Ric}_{M} \geq-(n-1) C^{2}$ is only used to bound the volume entropy of $M$ by $(n-1) C$. By [BCG3, there is an equivariant smooth map $\tilde{F}: \tilde{M} \rightarrow \tilde{N}$ such that

$$
\left|\operatorname{det}\left(\tilde{F}_{*}\right)\right| \leq\left(\frac{h(M)}{(n-1) \sqrt{|\lambda|}}\right)^{n} .
$$

From Theorem 1.1, we have

$$
\left|\operatorname{det}\left(\tilde{F}_{*}\right)\right| \leq\left(\frac{\sqrt{(n-1)|\lambda|}+k_{\Gamma}(\lambda, p)^{\frac{1}{2 p}}}{\sqrt{(n-1)|\lambda|}}\right)^{n}
$$

which completes the proof of Corollary 1.3(a). With Corollary 2.2, we can obtain Corollary 1.3(b). Precisely, if there is a continuous map from $M$ to $N$ of nonzero degree, then there is an equivariant smooth map $\tilde{F}: \tilde{M} \rightarrow \tilde{N}$ such that

$$
\begin{aligned}
\left|\operatorname{det}\left(\tilde{F}_{*}\right)\right| & \leq\left(\frac{h(M)}{h(N)}\right)^{n} \\
& \leq\left(\frac{(n-1) \sqrt{|\lambda|}+\sqrt{n-1} k_{\Gamma}(\lambda, p)^{\frac{1}{2 p}}}{h(N)}\right)^{n}<1
\end{aligned}
$$

since $K_{N}<-\left(\sqrt{|\lambda|}+\frac{k_{\Gamma}(\lambda, p)^{\frac{1}{2 p}}}{\sqrt{n-1}}\right)^{2}$. Then we have $\operatorname{vol}(N)<\operatorname{vol}(M)$, which is a contradiction to $\operatorname{vol}(M) \leq \operatorname{vol}(N)$.

\section{REFERENCES}

[B] W. Ballmann, Lectures on spaces of nonpositive curvature, Birkhäuser, 1995. MR.1377265 (97a:53053)

[BCG1] G. Besson, G. Courtois, S. Gallot, Entropies et rigidités des espaces localement symétriques de courbure strictement négative, Geom. Funct. Anal. 5 (1995), 731-799. MR.1354289 (96i:58136)

[BCG2] G. Besson, G. Courtois, S. Gallot, Minimal entropy and Mostow's rigidity theorems, Ergod. Theory \& Dynam. Sys. 16 (1996), 623-649. MR1406425 (97e:58177)

[BCG3] G. Besson, G. Courtois, S. Gallot, Real Schwarz lemma and some applications Rendiconti di Mathematica, Serie VII, vol. 18 Roma (1998), 381-410. MR1659814 (99k:53064)

[C] I. Chavel, Riemannian geometry-A modern introduction, Cambridge University Press, Cambridge, 2006. MR2229062 (2006m:53002)

[CD] C. Croke, N. Dairbekov, Lengths and volumes in Riemannian manifolds, Duke Math. J. 125 (2004), 1-14. MR2097355(2005k:53045)

[DS] N. Dairbekov, V. Sharafutdinov, Some problems of integral geometry on Anosov manifolds, Ergod. Theory \& Dynam. Sys. 23 (2003), 59-74. MR.1971196 (2005b:58053)

$[\mathrm{KH}] \quad$ A. Katok, B. Hasselblatt, Introduction to the modern theory of dynamical systems, Cambridge University Press, Cambridge, 1995. MR1326374 (96c:58055)

[G1] M. Gromov, Metric structures for Riemannian and non-Riemannian spaces, Birkhäuser, 1999. MR 1699320 (2000d:53065)

[G2] M. Gromov, Volume and bounded cohomology, IHES 56 (1982), 5-99. MR686042 (84h:53053)

[PW1] P. Petersen, G. Wei, Relative volume comparison with integral curvature bounds, Geom. Funct. Anal. 7 (1997), 1031-1045. MR1487753 (99c:53023)

[PW2] P. Petersen, G. Wei, Analysis and geometry on manifolds with integral Ricci curvature bounds II, Trans. Amer. Math. Soc. 353 (2001), 457-478. MR1709777(2002c:53055)

Department of Mathematics, Konkuk University, 1 Hwayang-dong, Gwanguin-gu, SeOul 143-701, Korea

E-mail address: shpaeng@konkuk.ac.kr 